\title{
PSGL-1 inhibits HIV-1 infection by restricting actin dynamics and sequestering HIV envelope proteins
}

\author{
Ying Liu', Yutong Song ${ }^{2}$, Siyu Zhang ${ }^{1}$, Min Diao ${ }^{3}$, Shanjin Huang ${ }^{3}$, Sai $\mathrm{Li}^{2}$ and Xu Tan (1)
}

\begin{abstract}
PSGL-1 has recently been identified as an HIV restriction factor that inhibits HIV DNA synthesis and more potently, virion infectivity. But the underlying mechanisms of these inhibitions are unknown. Here we show that PSGL-1 directly binds to cellular actin filaments (F-actin) to restrict actin dynamics, which leads to inhibition of HIV DNA synthesis. PSGL-1 is incorporated into nascent virions and restricts actin dynamics in the virions, which partially accounts for the inhibition of virion infectivity. More potently, PSGL-1 inhibits incorporation of Env proteins into nascent virions, causing a loss of envelope spikes on the virions as shown by Cryo-electron microscopy and super-resolution imaging. This loss is associated with a profound defect in viral entry. Mechanistically, PSGL-1 binds gp41 and sequesters gp41 at the plasma membrane, explaining the inhibition of Env incorporation in nascent virions. PSGL-1's dual anti-HIV mechanisms represent novel strategies of human cells to defend against HIV infection.
\end{abstract}

\section{Introduction}

HIV-1 infection redirects the cellular machinery towards mass production of new infectious virions, which renders the virus vulnerable to host defense strategies to undermine this production line $\mathrm{e}^{1-6}$. Currently we only have a very limited understanding of how the human innate immune system targets these vulnerabilities to restrict viral replication. For example, we do not know the functions and mechanisms of actions of the vast majority of the hundreds of potential antiviral genes upregulated by the interferons (interferon-stimulated genes, ISGs), the master regulators of antiviral defense ${ }^{7}$. HIV-1 restriction factors, including TRIM5 $\alpha$, APOBEC3, tetherin, SAMHD1, and SERINC3/SERINC5, are a small number of well-studied ISGs that block HIV-1 replication cycles at

\footnotetext{
Correspondence: Xu Tan (xutan@tsinghua.edu.cn)

${ }^{1}$ Beijing Advanced Innovation Center for Structural Biology, Beijing Frontier Research Center for Biological Structure, MOE Key Laboratory of Bioorganic Phosphorus Chemistry \& Chemical Biology, School of Pharmaceutical Sciences, Center for Infectious Disease Research, School of Medicine, Tsinghua University, Beijing 100084, China

${ }^{2}$ Beijing Advanced Innovation Center for Structural Biology, Beijing Frontier Research Center for Biological Structure, Tsinghua-Peking Center for Life Sciences, School of Life Sciences, Tsinghua University, Beijing 100084, China Full list of author information is available at the end of the article

These authors contributed equally: Ying Liu, Yutong Song
}

specific steps ${ }^{8}$. They employ a variety of mechanisms to exert potent restriction on the virus replication. On the other hand, HIV-1 has evolved countermeasures to evade from these blocks, often by inducing the ubiquitination and degradation or relocalization of restriction factors ${ }^{9}$. Given this reoccurring HIV-1-induced degradation of restriction factors, we conducted a genome-wide proteomic profiling of HIV-1 infection in primary human $\mathrm{CD}_{4}{ }^{+} \mathrm{T}$ cells and identified a new HIV-1 restriction factor, P-selectin glycoprotein ligand 1 (PSGL-1) ${ }^{10}$. PSGL1 inhibits HIV-1 reverse transcription and more potently, the infectivity of progeny virions ${ }^{10}$. Moreover, PSGL-1 is induced specifically by interferon $\gamma($ IFN- $\gamma$ ) and mediates the bulk of IFN- $\gamma^{\prime}$ s anti-HIV-1 activity in primary CD4 $4^{+}$ $\mathrm{T}$ cells ${ }^{10}$. To overcome this restriction, HIV-1 Vpu binds to PSGL-1 and recruits it to the E3 ligase, SCF ${ }^{\beta-T r C P 2}$ for ubiquitination and subsequent proteasomal degradation $^{10}$. Vpu-deficient HIV-1 has a significantly increased susceptibility to PSGL-1's infectivity inhibition ${ }^{10}$. Therefore, blocking the interaction between Vpu and PSGL-1 using small molecules could provide a potential therapeutic strategy to quench the infectivity of HIV-1. IFN- $\gamma$ has largely been considered as an immunomodulatory cytokine and its direct anti-HIV activity is starting to be 
appreciated $^{11}$. Our work demonstrated that PSGL-1 is a key mediator of IFN- $\gamma^{\prime}$ s antiviral activity in human $\mathrm{CD} 4^{+}$ $\mathrm{T}$ cells, the mechanism of which would therefore be important for the understanding of IFN- $\gamma$ 's role in the defense against HIV-1 infection.

PSGL-1 has been known as a leukocyte-specific singlepass transmembrane protein that mediates that adhesion of leukocytes to the endothelium at the inflammation sites $^{12,13}$. This adhesion is dependent on the binding of PSGL-1's extracellular domain to the cognizant selectin proteins (P-, E-, or L-selectins) expressed on the surface of endothelium and the attachment of PSGL-1's cytoplasmic domain $(\mathrm{CD})$ to the cortical actin cytoskeleton via ezrin and moesin proteins ${ }^{14,15}$. Here we show that the CD of PSGL-1 can directly bind actin filaments (F-actin) and inhibits F-actin disassembly catalyzed by the actin depolymerization protein cofilin $^{16}$. Consequently, PSGL-1 greatly reduces the actin assembly-disassembly dynamics, which are known be required for HIV-1 DNA synthesis $^{17}$ and the migration of HIV-1 DNA to the nucleus ${ }^{18}$. Remarkably, this restriction of actin dynamics is not only in the cells, but also in the released HIV-1 virions, which can partially account for the inhibition of virion infection by PSGL-1. Moreover, we observed that PSGL-1 inhibits the incorporation of HIV-1 envelope proteins into nascent virions, which accounts for the majority of the infectivity inhibition. Mechanistically, PSGL-1 binds gp41 protein and sequesters it at the plasma membrane, explaining the defect of envelope incorporation. Overall, PSGL-1's dual mechanisms of actions represent a novel antiviral defense strategy of ISGs.

\section{Results}

PSGL-1's modulation of F-actin intensity correlates with its anti-HIV-1 activity

PSGL-1 has been reported to attach to the cortical actin cytoskeleton by interacting with ezrin and moesin proteins that are linked to $\operatorname{actin}^{14,15}$. This interaction is dependent on the 66 amino-acid-long $\mathrm{CD}^{14,15}$. We confirmed that PSGL-1 can be co-immunoprecipitated with actin and this co-immunoprecipitation is abolished when the CD is deleted in Supplementary Fig. S1a. Fluorescence staining experiments demonstrated a clear colocalization of PSGL-1 and F-actin, with F-actin visualized either with the fluorescence tag-labeled phalloidin ${ }^{19}$ or with a GFP-tagged LifeAct reagent ${ }^{20}$ in Fig. 1a. We further observed that in Jurkat cells, overexpression of PSGL-1 led to an enhanced cortical F-actin intensity as shown by imaging (Fig. 1b, c) or by flow cytometry (Fig. 1d and Supplementary Fig. S1b). Consistent with the coimmunoprecipitation results, the CD of PSGL-1 is responsible for the effect on F-actin in Fig. 1d and Supplementary Fig. S1b. CRISPR/Cas9-mediated PSGL-1 knockdown in Jurkat cells led to decreased levels of
F-actin in Supplementary Fig. S1c, d. In addition, overexpression of PSGL-1 inhibits SDF-1-induced chemotaxis of Jurkat cells ${ }^{21}$ (Supplementary Fig. S1e), suggesting that PSGL-1 likely inhibits actin depolymerization and actin treadmilling, a driving force for chemotactic mobility of $\mathrm{T}$ cells. This is supported by the phalloidin staining of F-actin, which showed that PSGL-1 enhanced the basic level of F-actin intensity but inhibits the actin dynamics induced by SDF-1 in Supplementary Fig. S1f. One minute after SDF-1 treatment, Jurkat cells overexpressing luciferase control showed a rapid increase in F-actin density, consistent with a previous study ${ }^{18}$, but Jurkat cells overexpressing PSGL-1 do not have such an increase (Supplementary Fig. S1f).

We have previously shown that PSGL-1 is induced by IFN- $\gamma$ and mediates the anti-HIV-1 activity of IFN- $\gamma^{10}$. Consistently, IFN- $\gamma$ treatment increased F-actin intensity and this increase is largely abolished when PSGL-1 is knocked down by electroporation of siRNAs (Fig. 1e-g and Supplementary Fig. S1g). Importantly, F-actin intensity is negatively correlated with HIV-1 infection, suggesting that PSGL-1's anti-HIV-1 activity is associated with its modulation of F-actin intensity in Fig. 1e. We found that treating primary $\mathrm{CD} 4^{+} \mathrm{T}$ cells with an antibody that targets the extracellular domain of PSGL- $1^{22}$ can reduce F-actin intensity in the cells, likely due to a conformational coupling between the intracellular and extracellular domains in Fig. 1h. This reduced F-actin intensity due to antibody treatment is correlated with an increased HIV-1 infection in Fig. 1i, further supporting that PSGL-1 inhibits HIV-1 infection by modulating F-actin intensity.

\section{PSGL-1 inhibits F-actin depolymerization to restrict HIV-1 reverse transcription}

We have previously shown that PSGL-1 expressed in cells infected by HIV (target cells) can inhibit HIV-1 reverse transcription ${ }^{10}$. In addition, previous studies have demonstrated that actin cytoskeleton is required for HIV1 reverse transcription ${ }^{17}$, providing a potential link between PSGL-1's actin modulation and viral restriction. Indeed, we found that PSGL-1's inhibition of HIV-1 reverse transcription is dependent on the $\mathrm{CD}$ in Fig. 2a, b. Moreover, the $\mathrm{CD}$ itself is sufficient for the inhibition (Fig. 2b). We then performed a series of mutation experiments in the $C D$ and identified a highly conserved threonine (T393) as a key residue for the binding of PSGL-1 to actin (Fig. 2c, d and Supplementary Fig. 2). In contrast, mutations of two nearby conserved serine residues (S385 and S377) had no effect on the binding (Fig. 2c, d). Consistently, mutation of T393, but not mutations of S385 or S377, significantly abolished the inhibition of reverse transcription by PSGL-1 (Fig. 2d). In addition, PSGL-1 T393A mutant also lost the ability to increase F-actin 
a
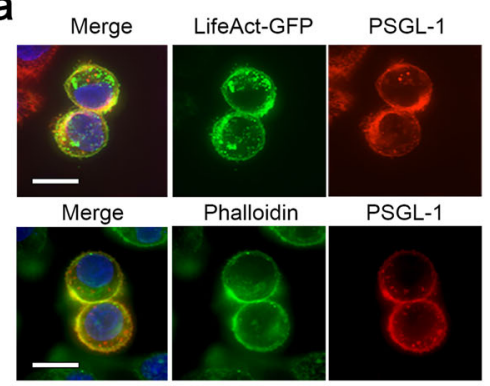

d

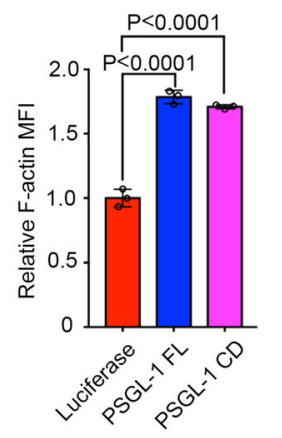

g

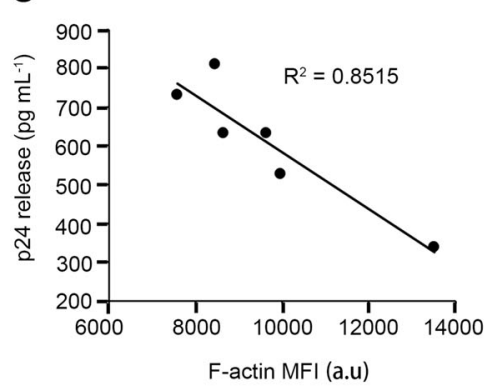

b

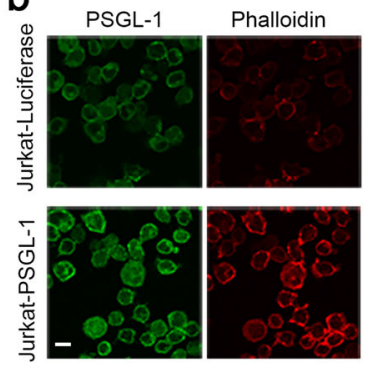

C

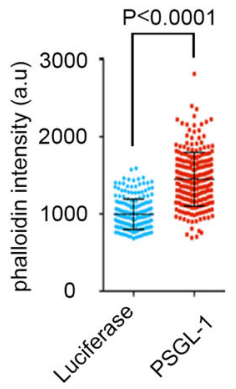

f

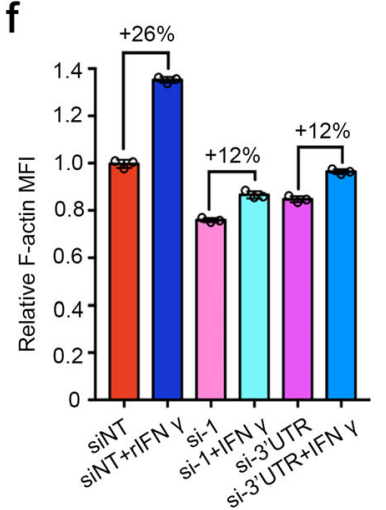

i

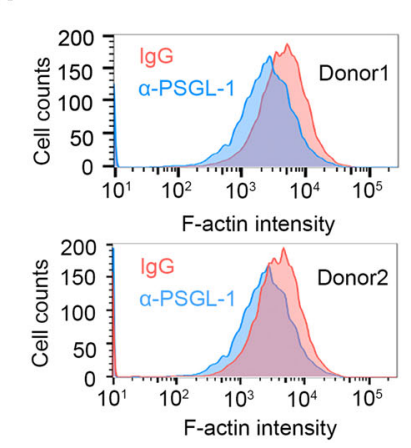

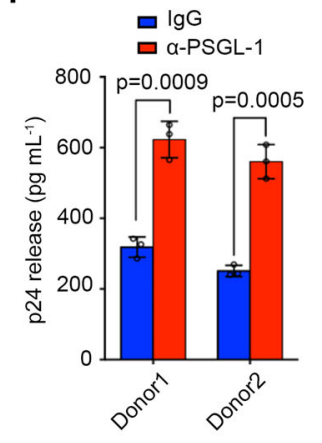

Fig. 1 PSGL-1 stabilizes cellular F-actin to restrict HIV infection. a Immunofluorescence staining of PSGL-1 in HeLa-based MAGI cells overexpressing PSGL-1 using anti-PSGL-1 antibody. Upper panel: PSGL-1 (red) colocalizes with LifeAct-GFP (green), which binds F-actin; Lower panel: PSGL-1 (red) colocalizes with phalloidin (green). Scale bar: $10 \mu \mathrm{m}$. b, c Immunofluorescence staining of PSGL-1 using anti-PSGL-1 antibody (green) and phalloidin (red) in Jurkat T cells overexpressing luciferase or PSGL-1. Scale bar: $5 \mu \mathrm{m}$. The phalloidin intensity of cells in each group is shown in c. $\mathbf{d}$ Jurkat T cells overexpressing luciferase, PSGL-1 or PSGL-1 CD (cytoplasmic domain) alone were stained with phalloidin and analyzed with FACS. Relative MFIs were normalized to luciferase group. MFI mean fluorescence intensity. $n=3$. e, $\mathbf{f}$ Activated primary CD4 ${ }^{+} \mathrm{T}$ cells were treated with IFN- $\gamma$ or mock-treated for $12 \mathrm{~h}$ before being electroporated with two different siRNAs targeting PSGL-1 or non-targeting control siRNA (siNT) for $48 \mathrm{~h}$. The cells were then either fixed for phalloidin staining and FACS quantification $(\mathbf{f})$ or infected with HIV-1 NL4-3 for $72 \mathrm{~h}$ before the supernatant being collected for p24 ELISA (e). $n=3$ for $\mathbf{e}, \mathbf{f}$. $\mathbf{g}$ Correlation between cellular F-actin intensities and HIV-1 infection rates in e, f. h, i Activated primary CD4 ${ }^{+}$ T cells from two healthy donors were incubated with PSGL-1 antibody or lgG for $2 \mathrm{~h}$ before being fixed for phalloidin staining (h) or infected with HIV-1 NL4-3 for $72 \mathrm{~h}$ before the supernatant being collected for p24 measurement (i). $n=3$.

intensity (Fig. 2e). These data support that actin binding and modulation of F-actin intensity by PSGL-1 are required for the reverse transcription inhibition.

Cortical F-actin intensity is determined by the relative speed of actin polymerization and depolymerization reactions in the cells ${ }^{23}$. Since PSGL-1 colocalizes with
F-actin, we suspected that it might increase the stability of F-actin by affecting actin depolymerization. We applied a well-established in vitro actin depolymerization assay using purified F-actin and cofilin protein, which is responsible for actin depolymerization ${ }^{24}$. In this assay, we first generated F-actin and then added purified cofilin to 


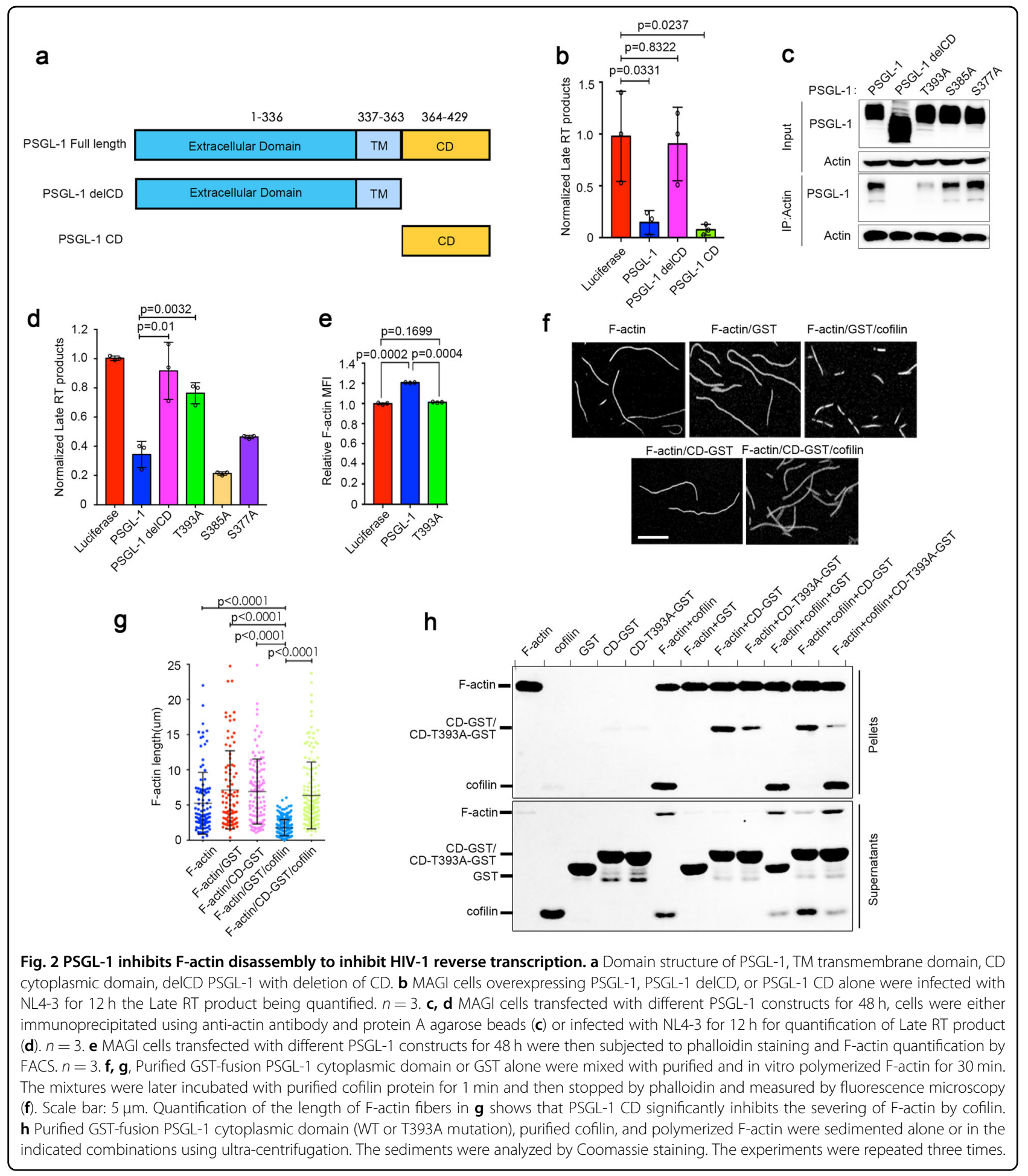

the F-actin to induce rapid depolymerization of the filaments, which can be observed by fluorescence imaging or by fluorescence TIRF microscopy in real time ${ }^{24}$. We also purified GST-tagged PSGL-1 cytoplasmic domain (CDGST), which is sufficient to inhibit HIV-1 reverse transcription, due to the difficulty to purify full-length membrane-associated PSGL-1. We found that CD-GST can prevent the depolymerization of F-actin by cofilin. As a control, GST alone had no effect on the depolymerization (Fig. 2f, g and Supplementary Movies S1, 2). We further utilized an F-actin co-sedimentation assay with purified proteins to show that: (1) CD-GST but not GST 
alone can be co-sedimented with F-actin; (2) T393A mutation significantly abolished the co-sedimentation of CD-GST with F-actin; (3) CD-GST but not T393A mutant significantly blocked the co-sedimentation of cofilin with F-actin in Fig. $2 \mathrm{~h}$. These in vitro data support that PSGL-1 competes with cofilin for binding to F-actin to block cofilin-mediated F-actin depolymerization, leading to an inhibition of HIV-1 reverse transcription.

\section{PSGL-1 increases F-actin intensity inside HIV-1 virions and affects virion infectivity}

We previously showed that PSGL-1 expressed in virus producing cells (producer cells) is packaged into nascent HIV-1 virions and potently restricts the infectivity of nascent virions even the virions are normalized by p24 levels in Fig. $3 a^{10}$. With a $\beta$-lactamase assay $(\mathrm{BlaM})^{25}$, we tested cellular entry of the virions packaged with PSGL-1 and found that PSGL-1 significantly inhibited viral entry of the nascent virions (Fig. 3b, c). The infectivity inhibition is largely correlated with entry inhibition (Fig. 3a, c). In addition, both the infectivity inhibition and the entry inhibition are dependent on the CD of PSGL-1 (Fig. 3a, c). We reasoned that this might be due to PSGL-1's effect on F-actin intensity since it has been shown that abundant actin incorporated inside the virions ${ }^{26}$. We confirmed that purified HIV-1 virions contain abundant actin and cofilin in Supplementary Fig. S3a. In addition, pre-treatment of the virions with actin polymerization inhibitors, latrunculin A or cytochalasin D promoted viral infection, supporting a role of actin inside the virions in the entry process (Fig. 3d). This increased infectivity is associated with increased viral entry as determined using the BlaM assay (Fig. 3e). It is worth noting that this promoting effect by actin modulators was not due to actin inhibitors' effects on the infected cells since treating the cells with the inhibitors at comparable concentrations (the concentration of the compounds in the cell media is much lower than the concentration in the virions due to the dilution of the virions when added to the cells) does not have a promoting effect on the infection (Supplemental Fig. S3b, c). Remarkably, the entry and infectivity promotions by these small molecule actin modulators were abolished in PSGL-1-containing virions (Fig. 3d, e), suggesting that PSGL-1 has an overriding effect on the actin network in the virions. Super-resolution imaging $(\mathrm{STORM})^{27}$ experiments show that virions packaged with PSGL-1 have significantly higher intensity of F-actin as specifically stained by phalloidin in Fig. 3f, g. Importantly, PSGL-1 T393A mutant, which is deficient for actin binding, caused little increase of F-actin intensity, suggesting that this increase is a specific effect associated with actin binding (Fig. 3f, g). To verify the STORM imaging results, we applied an ultra-centrifugation method to separate F-actin and actin monomers (G- actin). Western blotting of ultra-centrifugated virions demonstrated increased F-actin levels in virions packaged with PSGL-1, but not those packaged with PSGL-1 T393A mutant (Fig. 3h). This loss of ability to promote F-actin intensity of PSGL-1 T393A is associated with a reduction of infectivity inhibition and entry inhibition by this mutant compared with wild-type PSGL-1 (Fig. 3i, j), further supporting that PSGL-1's modulation of F-actin intensity inside virions contributes to the inhibition of virion infectivity.

PSGL-1 inhibits Env incorporation in virions and viral entry

It is worth noting that PSGL-1 T393A, which has little effect on F-actin intensity inside virions, can still strongly inhibit virion infectivity, albeit to a less degree than its wild-type counterpart (Fig. 3i, j). This suggests additional, more potent mechanism for the infectivity inhibition by PSGL-1. We found that the virions with PSGL-1 have much less Env proteins, namely gp41 and gp120, than control virions when normalized by p24 levels (Fig. 4a), which can explain the entry inhibition we observed earlier (Fig. 3b, c). On the other hand, HIV-1 infected Jurkat cells produced virions containing much more Env proteins if PSGL-1 had been knocked out using the CRISPR/ Cas9 system (Fig. 4b, c). This is true even the virions were normalized by p24, suggesting that PSGL-1's late effect on Env incorporation is a stronger effect than its early effect. This increase of Env proteins is more significant for $\mathrm{Vpu}$ deficient HIV than the wild-type virus, correlating with Vpu-mediated PSGL-1 degradation (Fig. 4b, c). Consistently, Vpu-deficient virions have a more significant increase of infectivity due to PSGL-1 knockout than the wild-type virus (Fig. 4d). In fact, we observed a very strong correlation between the gp41 level and virion infectivity in Fig. 4e, indicating that the infectivity block by PSGL-1 is due to the loss of Env proteins in the virions. Superresolution imaging showed that the level of gp41 protein in the virions is significantly increased if the virions were produced from Jurkat cells with PSGL-1 knocked out in Fig. 4f, g. On the contrary, if the producer cells overexpress PSGL-1, the gp41 protein levels would be significantly reduced as shown in Fig. 4h, i. Gag protein level is also decreased by PSGL-1, but to a less degree than that of gp41 (Fig. 4h, i). Consistent with viral infection and entry assays in Fig. 3a-c, this effect of PSGL-1 on gp41 level is abolished when CD is deleted (Fig. 4h, i), suggesting a requirement for the CD. We further applied cryo-electron microscopy (Cryo-EM) technique to directly visualize the virions, which showed that PSGL-1-containing virions have much less spikes on the surface of the virions compared with PSGL-1-free virions or PSGL-1 delCD containing virions, supporting an inhibition of Env incorporation in virions by PSGL-1 (Fig. 4j, k). 

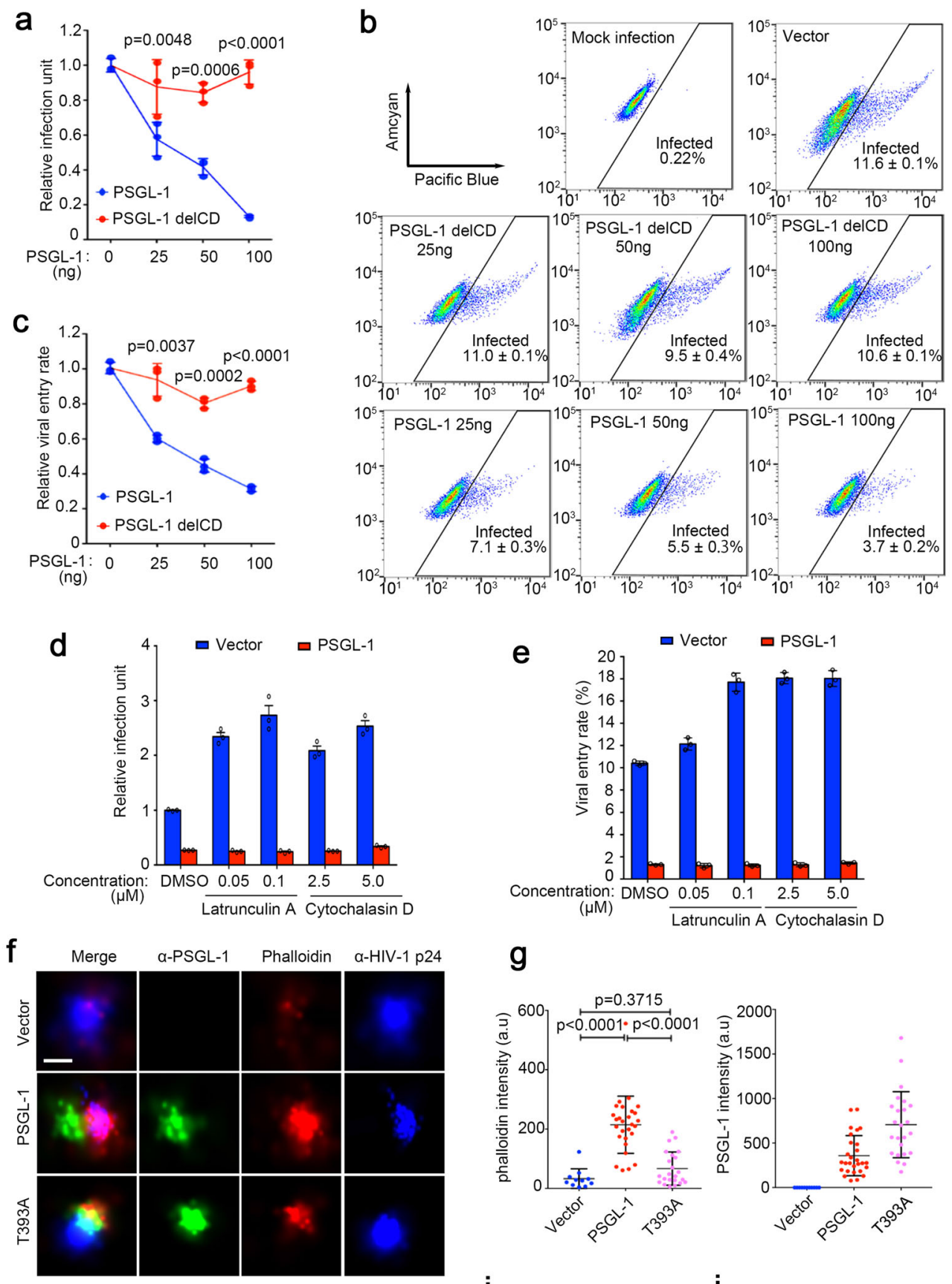

g

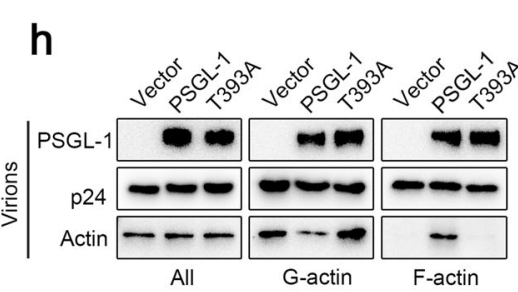

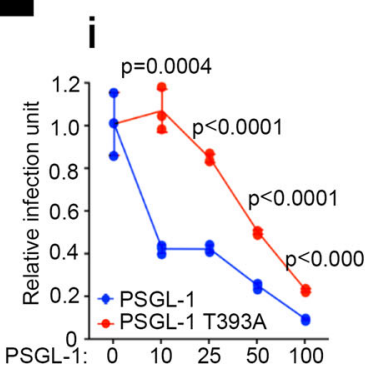

(ng)

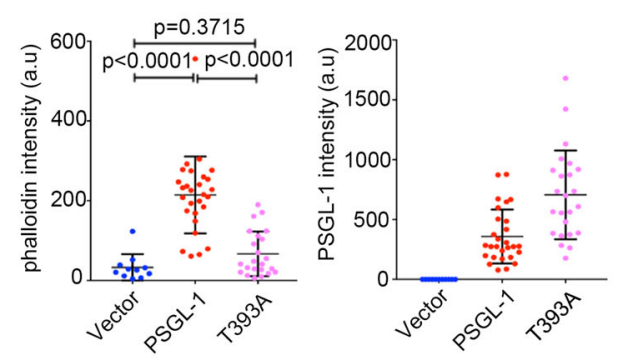

j

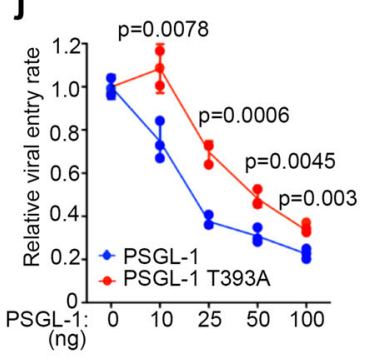

Fig. 3 (See legend on next page.) 
(see figure on previous page)

Fig. 3 PSGL-1 increases F-actin intensity inside HIV-1 virions and affects virion infectivity. a TZM-bl cells were infected with virions harvested from 293 T cells transfected with pNL4-3 and different amounts of plasmids expressing PSGL-1 and PSGL-1 delCD. Empty vector was used to normalize the total transfected DNA. The virions were normalized by p24 ELISA. The infection rates were quantitated with luciferase assay. $n=3$. b, c Jurkat cells were infected with Vpr-BlaM virions harvested from 293 T cells transfected with pNL4-3 and different amounts of plasmids expressing PSGL-1 and PSGL-1 deICD. Empty vector was used to normalize the total transfected DNA. The virions were normalized by P24 ELISA. The infection rates were measured by FACS. $n=3$. d NL4-3 virions packaged from 293 T cells with or without PSGL-1 overexpression were preincubated with indicated doses of F-actin inhibitors, latrunculin A, or cytochalasin D. TZM-bl cells were infected with treated virions for $48 \mathrm{~h}$ before the infection units were measured with luciferase assay. $n=3$. e Vpr-BlaM containing virions generated from 293 T cells with or without PSGL-1 overexpression were preincubated with latrunculin A or cytochalasin D at indicated concentrations for $2 \mathrm{~h}$. Jurkat cells were infected with the treated virions for $2 \mathrm{~h}$ and incubated with $\beta$-lactamase substrate overnight before being fixed and analyzed by FACS. $n=3$. f Virions harvested from producer 293 T cells transfected with PSGL-1 or PSGL-1 T393A or an empty vector were pelleted through 20\% sucrose cushion, fixed by $4 \%$ PFA and stained with antibodies and phalloidin before STORM imaging. Scale bar: $100 \mathrm{~nm}$. $\mathbf{g}$ Quantification of the intensities of F-actin or PSGL-1 staining in $\mathbf{f}$ shows that PSGL-1 but not PSGL-1 T393A significantly stabilizes F-actin in virions. $\mathbf{h}$ Virions containing PSGL-1 or PSGL-1 T393A were pelleted through 20\% sucrose cushion before being sedimented for fractions containing G-actin or F-actin and analyzed by Western blotting. i TZM-bl cells were infected with virions harvested from 293 T cells transfected with pNL4-3 and different amounts of plasmids expressing PSGL-1 and PSGL-1 T393A. Empty vector DNA was used to normalize the total DNA transfected. The infection rates were quantitated by luciferase assay. $n=3$. $\mathbf{j} V \mathrm{pr}$-BlaM containing virions generated from 293 T cells transfected with pNL4-3, Vpr-BlaM plasmid, and different amounts of PSGL-1 or PSGL-1 T393A plasmids. Empty vector was used to normalize the total DNA transfected. The viruses harvested were normalized by p24 ELISA and used to infect Jurkat cells for $2 \mathrm{~h}$. The cells were then incubated with $\beta$-lactamase substrate overnight before being fixed and analyzed by FACS. $n=3$.

\section{PSGL-1 interacts with gp41 and alters cellular localization of gp41}

To understand the effect of PSGL-1 on Env incorporation into virions, we first checked if it is due to a defect in the processing of gp160 into gp120 and gp41 using Western blot. The results showed no evidence of such a defect as the ratio of gp120 to gp160 is not altered by PSGL-1 (Supplementary Fig. S4). We then tested the interaction between gp41 and PSGL-1 since both are transmembrane proteins. Indeed, immunoprecipitation experiments using either protein as a bait showed that gp41 and PSGL-1 interacts with each other and deletion of $\mathrm{CD}$ abolished the interaction, consistent with the infectivity assays (Fig. 5a). Moreover, we found two highly conserved leucine residues (L368 and L369) in the CD (Supplementary Fig. S2) to be critical for the interaction between gp41 and PSGL-1 (Fig. 5a). Fluorescence staining experiments showed robust colocalization between gp41 and PSGL-1, supporting that the two proteins interact in the cells (Fig. 5b). Remarkably, PSGL-1 expression changed the cellular localization of gp41 from mostly intracellular and perinuclear localization to mostly plasma membrane localization (Fig. 5b, c). In contrast, PSGl-1 delCD and PSGL-1 LL/AA, both still membrane localized, largely lost the colocalization with gp41 and the ability to relocate gp41 from perinuclear localizations to the plasma membrane. In addition to the CXCR4-tropic NL43 strain, PSGL-1's inhibition of virus entry and effect on gp41 localization also apply to CCR5 strains such as YU2 and NL(AD8) (Supplementary Fig. S5a-d). These data suggest a model that PSGL-1 interacts with gp41 in a Cterminal domain-dependent fashion, which sequesters gp41 in the plasma membrane and inhibits its incorporation into nascent virions. Supporting this model,
PSGL-1 LL/AA, which cannot bind and relocate gp41, lost the ability to inhibit virion incorporation of Env proteins as shown by Western blotting, super-resolution imaging and Cryo-EM analysis (Fig. 5d and Supplementary Figs. S5a-d, S6a-d). Consistently, the infectivity inhibition of PSGL-1 LL/AA is also largely lost due to the mutations (Fig. 5e and Supplementary Fig. S5a-d). In comparison, the actin binding and F-actin promoting activity of PSGL$1 \mathrm{LL} / \mathrm{AA}$ remain unaffected (Supplementary Fig. S6e, f). In contrast to the important role of the LL motif, a mutation previously shown to affect PSGL-1 dimerization $(\mathrm{C} 310 \mathrm{~A})^{28}$ or triple mutations previously shown to affect PSGL-1's co-clustering with Gag (RRK 334/337/338 to AAA or 3A mutations) ${ }^{29}$ do not seem to have an effect on the infectivity inhibition of PSGL-1 (Supplementary Fig. S7). How does PSGL-1's interaction with gp41 excludes Env from being incorporated into nascent virions? A recent study showed that Env is first transported to the plasma membrane and then is endocytosed to the endosomal recycling compartment to assemble with Gag before being released. This trafficking was shown to be required for the viral incorporation of $\mathrm{Env}^{30}$. We applied the same Env construct incorporating fluorogen activating peptide tags, which allows pulse labeling of Env protein on the cell surface with a membrane impermeable fluorogen. Consistent with the previous report, we observed a rapid internalization of cell surface Env, while this internalization was inhibited by PSGL-1, which strongly colocalizes with Env on the cell membrane (Fig. 5f). In contrast, PSGL-1 delCD and PSGL-1 LL/AA are not able to inhibit this internalization. These data together support that a specific interaction between PSGL-1 and gp41 of Env is crucial to the infectivity inhibition by PSGL-1. 


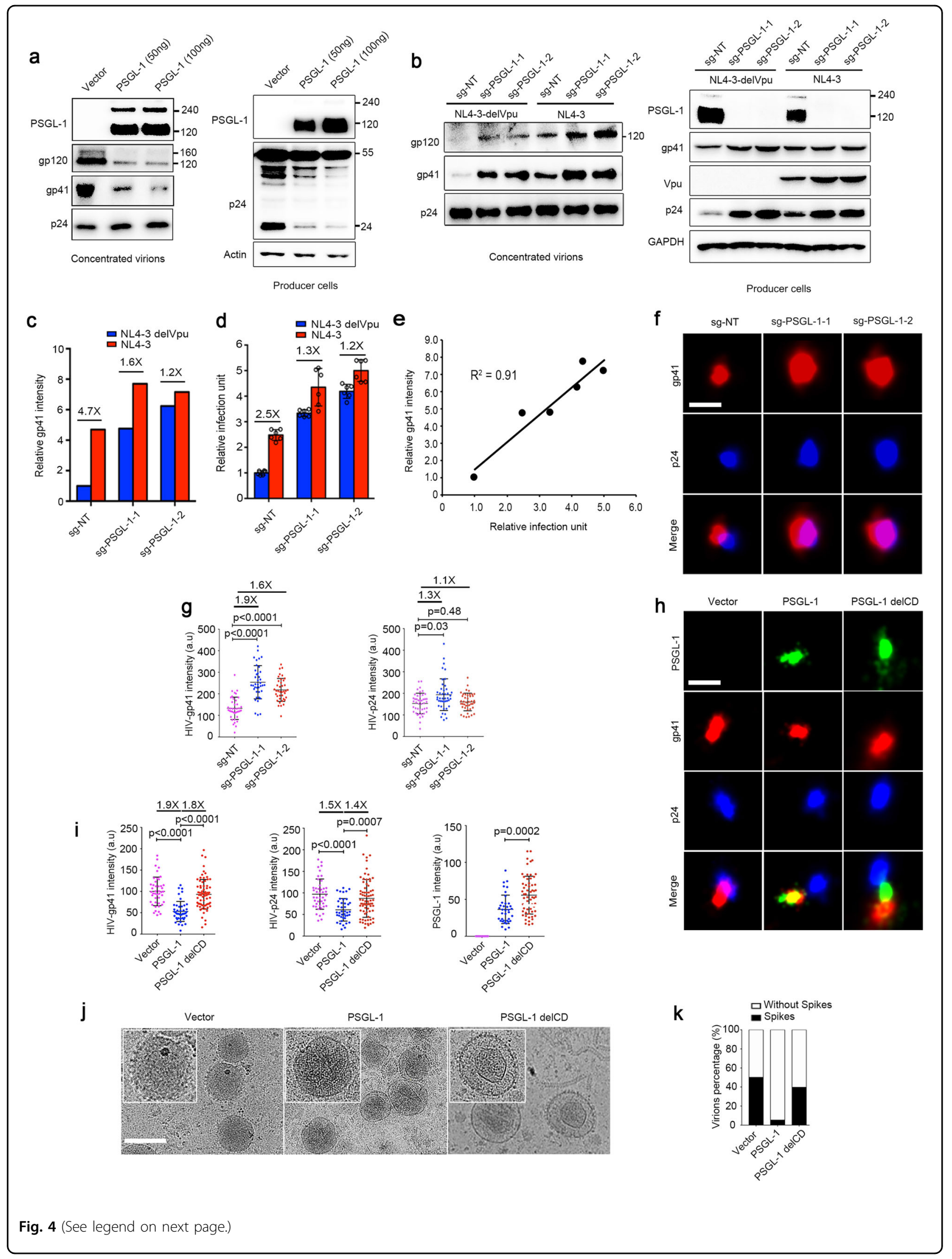


(see figure on previous page)

Fig. 4 PSGL-1 inhibits Env incorporation in virions and viral entry. a Virions harvested from producer 293 T cells transfected with two different doses of PSGL-1 or an empty vector were pelleted through $20 \%$ sucrose cushion were analyzed by Western blotting together with the cell lysates. b PSGL-1-knockout Jurkat cells lines with two different sgRNAs or a control cell line with a non-targeting (NT) sgRNA were infected with NL4-3 virus or NL4-3 delVpu virus. The supernatants containing newly released viruses were concentrated and analyzed by Western blotting. The cell lysates of the producer cells were also analyzed by Western blotting. c Quantification of the band intensity of gp41 in $\mathbf{b}$ normalized to the intensity of p24. The fold of change between NL4-3 virus and NL4-3 delVpu virus is shown for each cell group. $\mathbf{d}$ Virions harvested from infection experiments in $\mathbf{b}$ were normalized by p24 levels measured with ELISA and used to infect TZM-bl cells. The infectivity was measured by luciferase assays. The fold of change between NL4-3 virus and NL4-3 delVpu virus is shown for each cell group. e Correlation analysis between $\mathbf{c}$ and $\mathbf{d}$. $\mathbf{f}, \mathbf{g}$ Virions from PSGL-1 knockout or control Jurkat cell lines were collected after 5 days post infection with NL4-3 and pelleted through 20\% sucrose cushion were fixed and stained for STORM imaging. Representative images are shown in $\mathbf{f}$. Scale bar: $100 \mathrm{~nm}$. Quantification of STORM images results were showed in $\mathbf{g}$. The ratios between the average values of two groups and the $p$ values were shown. $\mathbf{h}, \mathbf{i}$ Virions from producer 293 T cells transfected with PSGL-1, PSGL-1 deICD, or an empty vector were pelleted through $20 \%$ sucrose cushion were fixed and stained for STORM imaging. Representative images are shown in $\mathbf{h}$. Scale bar: $100 \mathrm{~nm}$. Quantification of STORM images were showed in $\mathbf{i}$. The ratios between the average values of two groups and the $p$ values were shown. $\mathbf{j}, \mathbf{k}$ Concentrated virions were analyzed by cryo-EM analysis. Representative images were shown in $\mathbf{j}$ and quantification of images of virions shown in $\mathbf{k}$. Scale bar: $100 \mathrm{~nm}$.

\section{Discussion}

Here we demonstrate that PSGL-1 employs at least two different mechanisms to inhibit HIV infection. For the early inhibition of HIV DNA synthesis, PSGL-1 acts by binding with $\mathrm{F}$-actin and restricting cellular actin dynamics (Fig. 5g). A single mutation (T393A) of PSGL-1 that abolishes its F-actin binding also abolished its inhibition of HIV DNA synthesis. Consistently, IFN- $\gamma$ induces PSGL-1 expression and F-actin intensity, which is abolished by transfection of siRNAs targeting PSGL-1. The strong correlation between our in vitro actin depolymerization, binding assay and cellular infection experiments supports this actin restriction mechanism. Actin network has been well known be involved in HIV DNA synthesis ${ }^{17}$ and nuclear migration ${ }^{18}$, consistent with PSGL-1's restriction of these early processes by modulation of actin dynamics. Remarkably, this restriction also extends to actin in the virions. Actin has been reported as one of the most abundant proteins in HIV virions ${ }^{26}$. In addition, various actin interacting proteins such as ezrin, moesin, coronin-1A, and cofilin are all known to be present in the virions $^{26}$. However, the role of the actin cytoskeleton network in the virions has been elusive. We showed that one can promote viral infectivity by disrupting F-actin network with small molecules, while this promotion is abolished by PSGL-1 due to its overriding F-actin stabilizing activity. This observation suggests a role of actin cytoskeleton in viral entry, the mechanism of which is worth further investigation. PSGL-1 represents a novel restriction mechanism that targets the requirement of cytoskeleton for viral infection, which might be relevant to restriction of other types of viruses since actin dynamics are involved in the infection and pathogenesis of many different viruses ${ }^{31}$. PSGL-1's well-established function is mediating the adhesion of leukocytes at inflamed epithelium and T-cell migration ${ }^{12,13,32,33}$. Recently, PSGL-1 has been identified as a novel immune checkpoint molecule that mediates T-cell exhaustion ${ }^{34}$, it would be interesting to study the relationship between this immunoregulatory function and F-actin restriction of PSGL-1.

Compared with the inhibition of DNA synthesis, virion infectivity inhibition is a more potent block of HIV replication by PSGL-1. The bulk of this infectivity inhibition cannot be explained by PSGL-1's restriction of F-actin inside of virions. We found that PSGL-1 interacts with gp41 and sequesters it at the plasma membrane so that the nascent virions would be deficient of gp41 to form the spikes, which are required for efficient viral entry (Fig. 5e). Western blotting, super-resolution imaging, and Cryo-EM data all corroborate the significant decrease of Env proteins in the virions due to PSGL-1. Consistently, PSGL-1 significantly decreased the entry process in a BlaM assay. The infectivity inhibition is largely correlated with the entry inhibition. More importantly, we showed that PSGL-1 knockout in Jurkat cells promotes Env proteins incorporation into the nascent virions and enhances viral infectivity. These loss-of-function data ruled out the possibility of artifact due to protein overexpression. Structurally, PSGL-1's interaction with gp41 and its infectivity inhibition both require its C-terminal domain, particularly the LL motif in the domain, which supports a strong correlation between these two activities. Mechanically, how PSGL-1's interaction with gp41 excludes Env from being incorporated into nascent virions would require further investigation, which might shed light on our understanding of the process of viral assembly ${ }^{35}$. Our data support that PSGL-1's sequestration of gp41 at the plasma membrane would prevent further endocytosis and assembly of Env. In conclusion, PSGL-1 utilizes dual mechanisms to restrict HIV infection at early and late stages. Since the late inhibition of virion infectivity is more potent, HIV uses its Vpu protein to antagonize PSGL-1. Potentially anti-HIV drugs could be developed to inhibit Vpu's antagonism of PSGL-1 and incapacitate the virions. During the preparation and 


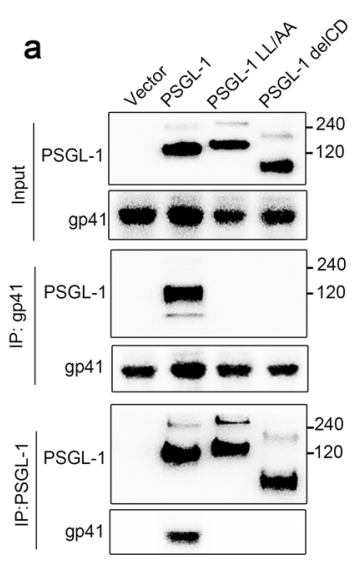

\section{b}

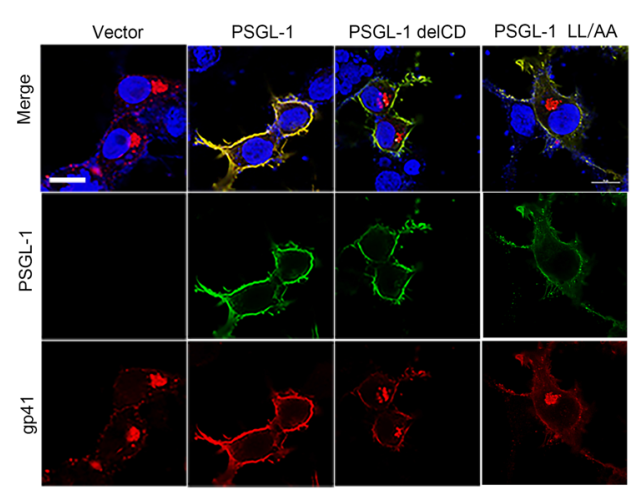

c
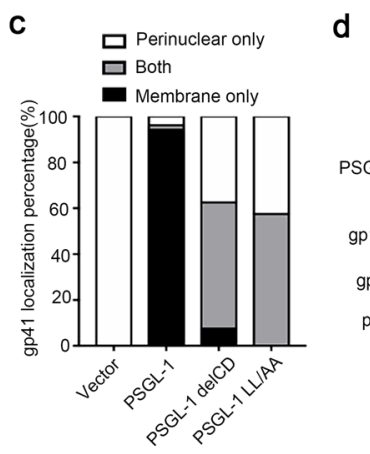

d

e
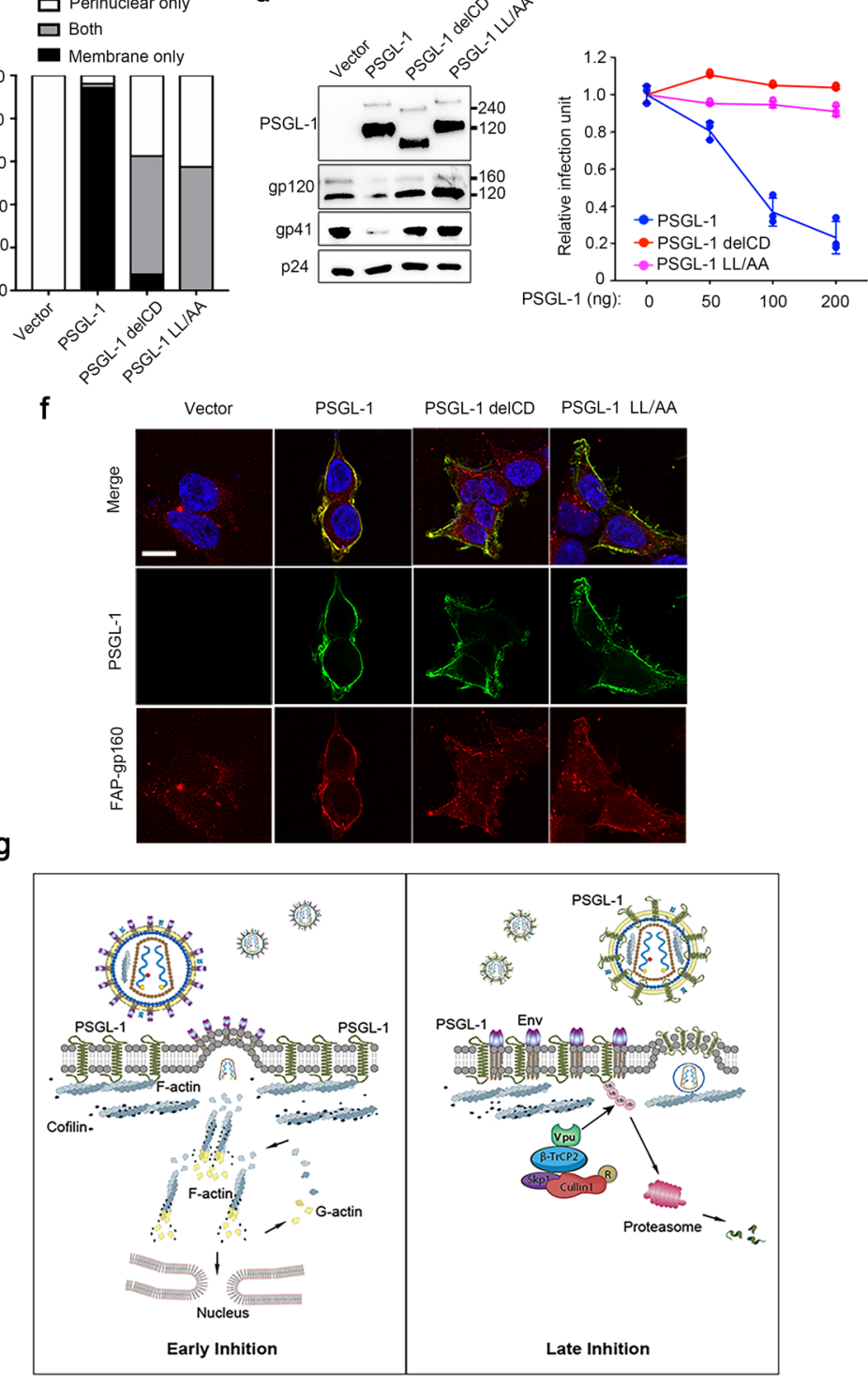

Fig. 5 (See legend on next page.) 
(see figure on previous page)

Fig. 5 PSGL-1 binds HIV Env and sequesters Env at the plasma membrane. a 293 T cells were separately transfected with pNL4-3 proviral plasmid or plasmids expressing PSGL-1, PSGL-1 delCD, PSGL-1 LL/AA, or an empty vector. One day after the transfection, PSGL-1 or gp41 were immunoprecipitated with protein A agarose beads with anti-PSGL-1 antibody or anti-gp41 antibody respectively and then the beads were mixed with cell lysates from transfection of the other protein in the IP. The cell lysates and the precipitated proteins were analyzed by Western blotting. b 293 T cells transfected with pNL4-3 and PSGL-1 or PSGL-1 deICD or PSGL-1 LL/AA or an empty vector were fixed and stained with anti-gp41 (red), anti-PSGL-1 (green) antibodies and DAPI (blue), and quantification of gp41 cellular localization of each sample is shown in c. Scale bar: $5 \mu \mathrm{m}$. $n=40$. d Virions harvested from producer 293 T cells transfected with PSGL-1 or PSGL-1 delCD or PSGL-1 LL/AA or an empty vector were pelleted through 20\% sucrose cushion were analyzed by Western blotting. e TZM-bl cells were infected with virions harvested from 293 T cells transfected with pNL4-3 and different amounts of plasmids expressing PSGL-1, PSGL-1 deICD, PSGL-1 LL/AA. Empty vector was used to normalize the total transfected DNA. The virions were normalized by p24 ELISA. The infection rates were quantitated with the luciferase assay. $n=3$. $\mathbf{f}$ Endocytosis of Env (gp160) protein in 293 T cells as visualized by pulse labeling of FAP-tag in the Env protein. 293 T cells were transfected with plasmids expressing PSGL-1, PSGL-1 deICD, PSGL-1 LL/AA and then stained with the fluorogen MG-11p (red) for 5 min. Cells were fixed after a 20-min chase period and stained with an anti-PSGL-1 (green) antibody and DAPI (blue). Scale bar: $5 \mu \mathrm{m}$. $\mathbf{g}$ Model of the mechanisms of the anti-HIV activity of PSGL-1. In the early stage of HIV life cycle, PSGL-1 restricts cortical actin filament to inhibit HIV reverse transcription. In the late stage of the life cycle, PSGL-1 prevents envelope incorporation into the nascent virions and blocks viral infectivity. PSGL-1 also stabilize F-actin inside of nascent virions to affect their infectivity.

review of this study, two papers reported mechanistic studies on PSGL-1's anti-HIV functions ${ }^{36,37}$. These studies proposed a model emphasizing the physical role of virion-incorporated PSGL-1 in occluding virions from attachment to the target cells. Since different study systems, cell lines, and different protein expression levels were used in the three studies, further investigation is warranted to incorporate and clarify these findings.

\section{Materials and methods \\ Cells and cell culture}

A total of 293T cells (from ATCC), TZM-bl, and MAGI cells (from NIH AIDS Reagents Repository) were cultured in DMEM supplemented with $10 \%$ heat-inactivated FBS, $6 \mathrm{mM}$ L-glutamine, and penicillin and streptavidin. Jurkat E6.1 cells were cultured in RPMI-1640 containing 10\% FBS, $6 \mathrm{mM}$ L-glutamine, and Pen/Strep. Peripheral blood mononuclear cells (PBMCs) were isolated from healthy human donors using Ficoll-Paque PLUS (GE Healthcare). CD4 $\mathrm{T}$ cells were purified from PBMCs using anti-human CD4+ magnetic beads (Miltenyi) and then cultured in RPMI supplemented with $10 \%$ heat-inactivated FBS, $\beta$-mercaptoethanol, and $6 \mathrm{mM}$ L-glutamine. Naïve CD4 T Cells were activated with CD3/CD8 beads (Invitrogen) and human recombinant IL-2 (30 U/ml, Roche) for $72 \mathrm{~h}$.

\section{Plasmids, transfection, and viral particle production}

For exogenous expression in mammalian cells, human PSGL-1 or its truncated forms and luciferase were cloned in a pLenti-CMV vector with $\mathrm{N}$-terminal $\mathrm{HA}$ tag and FLAG tag, or a PLX-304 vector with a C-terminal V5 tag. The vectors were transfected into cell lines using Neofect transfection reagent following the manufacturer's protocol (Neofect Biotech, Beijing). For protein purification, human PSGL-1 cytoplasmic domain was cloned in pGEX expression vector with C-terminal GST tag and human cofilin was cloned in pET expression vector with a Cterminal His tag. pCMV-LifeAct-GFP plasmid was purchased from Ibidi. NL4-3 viral particles were produced by transfection of 293T in a T75 flask with $20 \mu \mathrm{g}$ pNL4-3. SgRNA-expressing and ORF- expressing plasmids were packaged into lentiviral particles in each well of a six-well plate with $10 \mu \mathrm{g}$ PLKO.1, lentiCRISPR v2, or pLX304 or pCMV backbone vectors, $2 \mu \mathrm{g}$ pCG-VSV-G, $1 \mu \mathrm{g}$ pCMVTat, $1 \mu \mathrm{g}$ pCMV-Rev, and $1 \mu \mathrm{g}$ pCMV-Gagpol. For packaging of Vpr-BlaM viral particles, $10 \mu \mathrm{g}$ pNL4-3, $3.4 \mu \mathrm{g}$ pMM310-Vpr-BlaM, and $1.7 \mu \mathrm{g}$ pAdvantage were transfected into each well of a six-well plate. Twenty-four hours afterAfter $24 \mathrm{~h}$ of transfection, the medium was removed. Viral supernatants were collected after another $2424 \mathrm{~h}$ and filtered at $0.45 \mu \mathrm{M}$.

\section{sgRNA and siRNA}

All the siRNAs were purchased from GenePharma, Shanghai. For siRNA nucleofection, the siRNAs were transfected into human CD4+ T cells using the Amaxa Nucleofector following the manufacturer's protocols (Lonza). The siRNAs used in this study were purchased from GenePharma and the sequences are as below:

siNT sense: UUCUCCGAACGUGUCACGUTT siNT antisense: ACGUGACACGUUCGGAGAATT siPSGL-1-1 sense: GCCACUAUCUUCUUCGUGUTT siPSGL-1-1 antisense: ACACGAAGAAGAUAGUGG CTT

siPSGL-1-3' UTR sense: CAGGAGGCCAUUUACU UGATT

siPSGL-1-3' UTR antisense: UCAAGUAAAUGGCCU CCUGTT

The sgRNA sequences used in this study: sgNT (Non-targeting control) TTTGAAGTATGCCT CAAGGT sgPSGL-1-2 ATCTAGGTACTCATATTCGG 


\section{Real-time PCR amplification}

For the measurement of viral late RT products and 2LTR circles, the following primers were used for a TaqMan based qRT-PCR ${ }^{38}$ :

Late RT forward primer, 5'-TGTGTGCCCGTCT GTTGTGT-3'

Late RT reverse primer, $5^{\prime}$-GAGTCCTGCGTCGAGAG AGC-3'

Late RT probe, 5'-(FAM)- CAGTGGCGCCCGAACA GGGA-3'

2-LTR forward primer, $5^{\prime}$-AACTAGGGAACCCACT GCTTAAG-3'

2-LTR reverse primer, 5'-TCCACAGATCAAGGATA TCTTGTC-3'

2-LTR probe, $5^{\prime}$-(FAM)-ACACTACTTGAAGCACTC AAG-3'

Mitochondrial forward primer, 5'-ACC- CACTCC CTCTTAGCCAATATT-3'

Mitochondrial reverse primer, 5'-GTAGGGCTAGG CCCACCG-3'

Mitochondrial probe, $5^{\prime}$-(TET) CTAGTCTTTGCCGC CTGCGAAGCA (TAMRA)-3'

\section{Antibodies and beads}

The following antibodies were used for cell cytometry, immunostaining, or western blotting: anti-PSGL-1 (Santa Cruz Biotech, sc-13535, 1:200), anti-cofilin (Cell Signaling, 5175, 1:500), anti-p24 (NIH AIDS Reagent Program, 1513, 1:1000), anti- Actin (sc-8432, 1:1000), anti-GAPDH (ZSGB-Bio, TA-08, 1:1000), anti-gp41(NIH AIDS Reagent Program, 1242, 1:500), anti-gp120(Abcam, ab21179). For IP and pull-down assay, protein A-agarose beads (Santa Cruz, sc-2001; Bimake, B23202) were used. The fluorescent secondary antibodies used in this study were Alexa Fluor 568 goat anti-human IgG(H+L) (Molecular Probes, A-21090, 1:1000), Alexa Fluor 488 goat anti- mouse IgG $(\mathrm{H}+\mathrm{L})$ (Molecular Probes®, A-11001, 1:1000), Alexa Fluor 594 goat anti- mouse IgG(H+L) (Molecular Probes, A-11005, 1:1000), Alexa Fluor 568 goat anti-rabbit $\operatorname{IgG}(\mathrm{H}+\mathrm{L})$ (Molecular Probes, A-11010, 1:1000), Alexa Fluor 647 goat anti-rabbit IgG(H+L) (Molecular Probes, A-21245, 1:1000), Alexa Fluor 647 rabbit anti-goat IgG(H $+\mathrm{L}$ ) (Molecular Probes, A-32849, 1:1000). For F-actin staining, Alexa Fluor 568 Phalloidin (Molecular Probes, A-12380, 1:500) was used.

\section{Immunoprecipitation assay}

For actin IP assay, MAGI cells seeded in $6 \mathrm{~cm}^{2}$ plates were transfected with $5 \mu \mathrm{g}$ pCMV-PSGL-1 or pCMVPSGL-1 delCD, respectively. The cells were lysed with RIPA buffer (50 mM Tris-Cl pH7.4, $150 \mathrm{mM} \mathrm{NaCl}, 1 \%$ NP-40, $0.5 \%$ sodium deoxycholate, $0.1 \%$ SDS) plus protease inhibitor cocktail (Bimake, B14001) $48 \mathrm{~h}$ after transfection. Cell lysates were incubated with Actin antibody at $4{ }^{\circ} \mathrm{C}$ overnight. The mixtures were then incubated with $40 \mu \mathrm{L}$ agarose beads at $4{ }^{\circ} \mathrm{C}$ for $2 \mathrm{~h}$. The beads were washed three times the next day and boiled in 1X SDS-PAGE buffer at $9595^{\circ} \mathrm{C}$ for $10 \mathrm{~min}$. For gp41 IP assay. 293T cells in each well of six-well- plates were transfected with $2 \mu \mathrm{g}$ pNL4-3, pCMV-empty vector, pCMV-PSGL-1, pCMV-PSGL-1 delCD individually. The cells were lysed with binding buffer $(50 \mathrm{mM}$ Tris-Cl $\mathrm{pH} 7.5,150 \mathrm{mM} \mathrm{NaCl}, 0.5 \% \mathrm{NP}-40$ ) plus protease inhibitor cocktail at $48 \mathrm{~h}$ after transfection. NL4-3-overexpressing 293T cell lysate was mixed with cell lysate of cells expressing PSGL-1, PSGL-1 delCD, or pCMV-empty vector and incubated with $40 \mu \mathrm{l}$ agarose beads and antibodies following the Bimake agarose beads protocol.

\section{Flow cytometry}

For the SDF-1 stimulation, a total of $2 \times 10^{5}$ Jurkat cells were stimulated with SDF-1 $(400 \mathrm{ng} / \mathrm{ml}$, dissolved in sterilized water) at $37^{\circ} \mathrm{C}$. The cells were then fixed quickly using $500 \mu \mathrm{L}$ BD CytoPerm/Cytofix Solution (BD554722) for $20 \mathrm{~min}$ at room temperature, washed twice with cold Perm/Wash buffer (BD554723), and stained with Alexa Fluor 568 -conjugated phalloidin (Molecular Probes, A12380) for $30 \mathrm{~min}$ at $4{ }^{\circ} \mathrm{C}$ in the dark. The cells were then washed twice and resuspended in $2 \%$ paraformaldehyde in PBS and analyzed on a Becton Dickinson LS5R Fortessa flow cytometer. For the antibodies pre-treat experiment, cells were pre-incubated with antibodies at indicated concentrations for $2 \mathrm{~h}$ at $4{ }^{\circ} \mathrm{C}$. Then the cells were fixed and stained with Alexa Fluor 568 Phalloidin.

\section{Transwell assay for chemotaxis}

A total of $5 \times 10^{5}$ to $1 \times 10^{6}$ PSGL-1-overexpressing or Firefly luciferase-expressing Jurkat cells were added to the upper chamber of transwell slide (Costar 3422, Corning), $500 \mu \mathrm{L} \mathrm{RPMI} / \mathrm{FBS}+20 \mathrm{ng} / \mathrm{ml} \mathrm{SDF}-1$ was added to the lower chamber, and cells were allowed to migrate for $2 \mathrm{~h}$ at $3737^{\circ} \mathrm{C}$ in an incubator. Cell numbers were primarily counted with a Bio-Rad TC10 cell counter and then analyzed on a Becton Dickinson LSR Fortessa flow cytometer.

\section{BlaM assay}

Vpr-BlaM NL4-3 was produced as previously described $^{39}$. For infection of Jurkat cells, a total of $4 \times 10^{5}$ Jurkat cells in each well of 12-well plates were incubated with NL4-3 virus for $2 \mathrm{~h}$ at $37^{\circ} \mathrm{C}$. The cells were washed three times with PBS, and then $0.2 \mathrm{~mL} 1 \times$ CCF4-AM dye solution (Life Technologies, K1095) in phenol-free DMEM/HEPES/2\% FBS was added to the cells. The cells were incubated at $11^{\circ} \mathrm{C}$ overnight, then washed twice with PBS, fixed in $2 \%$ paraformaldehyde in PBS for $20 \mathrm{~min}$ in dark, and analyzed on a Becton Dickinson LSR Fortessa flow cytometer (Fluorescence channels: Pacific Blue and AmCyan). 


\section{Confocal microscopy}

For F-actin intensity measurement in Jurkat E6.1 cells, $4 \times 10^{5}$ cells were fixed and stained with anti-PSGL-1 antibody (Santa Cruz Biotech, sc-13535, 1:200) and Alexa Fluor 568 Phalloidin (Molecular Probes, A-12380, 1:500) as previously described. Fixed cells were suspended in $200 \mu \mathrm{L}$ PBS and dried in a clean cover glass. The cells were analyzed on an ArrayScan VTI 700 (Thermo Scientific, Fluorescence channels: PE and FITC). For F-actinPSGL-1 colocalization experiment, MAGI cells in 12-well plate were transfected with $1 \mu \mathrm{g}$ pLifeAct-GFP and $1 \mu \mathrm{g}$ pCMV-PSGL-1 or pCMV-PSGL-1 alone, respectively. Cells were fixed and stained with antibodies. For the gp41-PSGL-1 colocalization experiment, 293T cells in 12well -plate were transfected with $1 \mu \mathrm{g}$ pNL4-3 and $1 \mu \mathrm{g}$ pCMV vector or PSGL-1 constructions. Cells were fixed $20 \mathrm{~h}$ post infection and stained with PSGL-1 antibody (Santa Cruz Biotech, sc-13535, 1:200), gp41 antibody (NIH AIDS Reagent Program, 1412, 1:500) and imaged as described above. For phalloidin staining, stained cells were imaged using an Olympus FV1200 Confocal Microscope with a 40 NA 0.95 or a 60 NA 1.3 oil objective. For gp41-PSGL-1 colocalization, cells were imaged using Nikon A1R HD25 microscope with a 100 NA 1.3 oil objective. Samples were excited with $480 \mathrm{~nm}$ for GFP or $543 \mathrm{~nm}$ for Alexa 568 and 594 or $640 \mathrm{~nm}$ for Alexa 647 and $405 \mathrm{~nm}$ for DAPI. F-actin intensities were measured by Imaris software. For the FAP-Env labelling experiment, 293T cells were transfected with FAP-gp160 (kindly provided by Dr. Paul Spearman) and plasmids expressing PSGL-1 constructions at a ratio of 2:1 in 24-well plate. The cells were exposed to $200 \mathrm{nM}$ MG-11p (Spectragenetics) for $5 \mathrm{~min}$ in dark and then washed with PBS. Then the cells were incubated with DMEM for 20 min at $37^{\circ} \mathrm{C}$ and then fixed with PFA and stained with antiPSGL-1 antibody for imaging analysis.

\section{STORM image acquisition and analysis}

Purified HIV particles were fixed using by BD CytoPerm/Cytofix Solution and adhered to poly-L-lysine solution (SIAMG, P4832) coated glass cover slips for $30 \mathrm{~min}$ at room temperature. Cover slips were then blocked using 2\% BSA/PBS for $20 \mathrm{~min}$. Particles were stained for PSGL-1 antibody, gp41 antibody and p24 antibody and Alexa Fluor 568- conjugated- anti-human, Alexa Fluor 488- conjugated- anti-mouse and Alexa Fluor 647- anti-goat secondary antibody or Alexa Fluor 568 phalloidin as indicated. Following immunostaining, particles were washed with PBS and overlaid with image solutions plus 2-mercaptoethanol following Nikon NSTORM manual and imaged using N-STORM microscopy. Images were exported and fluorescence of each channel were measured by Nikon NIS-Elements AR Analyzer.

\section{Inhibitor treatments of cells}

For the SDF- 1 treatment, $50 \mathrm{ng} / \mathrm{mL}$ SDF-1 were added into medium for $1 \mathrm{~min}$ before Jurkat cells being fixed and stained with phalloidin. For the actin inhibitor treatments of viral particles, supernatants from 293T cells transfected with pNL4-3 with pCMV-vector or pCMV-PSGL-1 were pre-incubated with latrunculin A or DMSO control for 2 $\mathrm{h}$ at $37^{\circ} \mathrm{C}$ or pre-incubated with cytochalasin $\mathrm{D}$ at $20 \mathrm{~min}$ at $37^{\circ} \mathrm{C}$ before being added into cells. The infection rates were determined using luciferase assay or BlaM assay.

\section{p24 ELISA}

For the virus packaged by $293 \mathrm{~T}$ cells in 12-well plate, cells were transfected with $1 \mu \mathrm{g}$ pNL4-3 proviral plasmids and different doses of plasmids expressing PSGL-1, PSGL1 delCD, or PSGL-1 T393A. After $8 \mathrm{~h}$ of transfection, cells were washed twice and the medium were replaced. Two days post transfection, the supernatant was collected for p24 measurement using a commercial p24 ELISA kit (Sinobiological). For the primary CD4+ T cells infection, $1 \times 106$ activated CD4 $+\mathrm{T}$ cells were stimulated by IFN- $\gamma$ for $24 \mathrm{~h}$ before being transfected with 3 siRNAs following Amaxa Nucleofector following the manufacturer's protocols (Lonza). Two days post transfection, the cells were pre-treated with antibodies for $2 \mathrm{~h}$ at $37^{\circ} \mathrm{C}$. Then the cells were infected with NL4-3 virus for $6 \mathrm{~h}$, then wash three times with PBS. The supernatant was collected for p24 measurement $72 \mathrm{~h}$ post infection.

\section{Firefly luciferase assays}

Cells transfected with pNL4-3 proviral plasmids and plasmids expressing PSGL-1 or PSGL-1 truncations as described above. Supernatant were collected $48 \mathrm{~h}$ after transfection and the virus titrations were measured by p24 ELISA kit. Then TZM-bl cells in 12-well plate were infected with supernatant with equal titration from each group of 293T cells. At $48 \mathrm{~h}$ post-infection cells were lysed in cell culture lysis buffer. Virus infectivity was assayed by measuring chemiluminescent firefly luciferase activity using the commercial kit from Promega.

\section{Protein purification}

The PSGL-1 CD- and cofilin-expressing plasmids were used to transform competent E. coli BL21 strain, which was then grown in LB medium to an OD600 of 0.8 and induced with $0.5 \mathrm{mM}$ IPTC at $16^{\circ} \mathrm{C}$ overnight. The harvested cells were sonicated in lysis buffer $(50 \mathrm{mM}$ Tris $\mathrm{HCl}$ $\mathrm{pH} 7.5,150 \mathrm{mM} \mathrm{NaCl}, 2 \mathrm{mM}$ dithiothreitol, and $100 \mathrm{nM}$ PMSF). The soluble fraction of cell lysate was purified with the Ni-NTA resin or GST resin (GE) and further fractionated by ion-exchange chromatography (Source Q, GE) and size exclusion chromatography (SD75, GE). Every fraction was collected and validated by SDS-PAGE before the confirmed fractions were combined for later use. 


\section{Fluorescence microscopy of actin filaments}

This experiment protocol was as described by Zheng et al. ${ }^{40}$. Briefly, $12 \mu \mathrm{M}$ actin was incubated at room temperature for $30 \mathrm{~min}$ in polymerization buffer $(10 \mathrm{mM}$ imidazole, $0.2 \mathrm{mM} \mathrm{CaCl}$, $\mathrm{pH} 7.0,2 \mathrm{mM} \mathrm{MgCl} 2,50 \mathrm{mM}$ $\mathrm{KCl}, 0.2 \mathrm{mM}$ ATP, $1 \mathrm{mM}$ EGTA, $0.5 \mathrm{mM}$ DTT, and 3 $\mathrm{mM} \mathrm{NaN3}$ ) and with $40 \mu \mathrm{M}$ GST or PSGL-1 CD-GST or buffer only. The resulting actin filaments $(4 \mu \mathrm{M})$ were then incubated with $10 \mu \mathrm{M}$ cofilin for $1 \mathrm{~min}$. An equimolar amount of phalloidin was then added to label actin filaments and stop the reaction. Finally, actin filaments were diluted to $10 \mathrm{nM}$ with fluorescence imaging buffer (10 mM imidazole- $\mathrm{HCl}, \mathrm{pH} 7.0,50 \mathrm{mM} \mathrm{KCl}, 1 \mathrm{mM}$ $\mathrm{MgCl} 2,100 \mathrm{mM}$ DTT, $100 \mathrm{mg} / \mathrm{mL}$ Glc oxidase, $15 \mathrm{mg} /$ $\mathrm{mL}$ Glc, $20 \mathrm{mg} / \mathrm{mL}$ catalase, and $0.5 \%$ methylcellulose). A sample of $2.5 \mu \mathrm{L}$ of actin filament mixture was then placed on a poly-L-Lys-coated cover slip and imaged with a $60 \times$ oil objective. Image were captured with Image-Pro Express 6.3 software. The length of individual actin filaments was quantified by ImageJ.

\section{Direct visualization of actin filament severing and depolymerization by TIRF microscopy}

This experiment protocol was as described by Zheng et $\mathrm{al}^{40}$. Briefly $1.5 \mu \mathrm{M}$ polymerized actin labeled with 5(and-6)-carboxytetramethylrhodamine- succinimidylester were incubated with $50 \mu \mathrm{M}$ GST or PSGL-1 CD-GST for $30 \mathrm{~min}$ and then injected into the flow cell coated with 25 nM N-ethylmaleimidemyosin. Cofilin was injected into the chamber at $0.2 \mu \mathrm{M}$ final concentration. Single actin filaments were observed by TIRF illumination with an Olympus IX81 microscope equipped with a 100x oil objective (1.49 numerical aperture). Images were collected for $5 \mathrm{~min}$ with an interval of $1 \mathrm{~s}$ using a Photometrics cascade II 512 CCD camera (Major Instruments) with Micromanager software.

\section{Actin co-sedimentation assay}

Actin was polymerized as described above. For the F-actin- binding complex containingcotainining $10 \mu \mathrm{M}$ cofilin, $30 \mu \mathrm{M}$ GST, CD-GST or CDT393A-GST and $10 \mu \mathrm{M}$ F-actin filaments in KMEI buffer (10× KEI buffer: $500 \mathrm{mM} \mathrm{KCl}, 100 \mathrm{mM}$ imidazole $\mathrm{pH}$ 7.0, $10 \mathrm{mM} \mathrm{MgCl}$, $10 \mathrm{mM}$ EGTA) at $30^{\circ} \mathrm{C}$ for $1 \mathrm{~h}$, and then centrifuged at $10,000 \mathrm{~g}$ for $30 \mathrm{~min}$ at $4{ }^{\circ} \mathrm{C}$. They supernatant and pellet were separated and boiled for $10 \mathrm{~min}$, followed by electrophoresis and coomassie staining. This experiment was following protocol described by Doolittle et $\mathrm{al}^{41}$.

\section{F-actin isolation}

F-actin isolation protocol was followed by Yang et $\mathrm{al}^{42}$. Briefly, viruses were filtered through $0.45 \mu \mathrm{m}$ filters and pelleted through $20 \%$ sucrose cushion and then lysed in actin stabilization buffer (100 mM Pipes, pH 6.9, 5\% glycerol, 5 mM MgCl2, 5 mM EGTA, 1\% Triton X-100, 1 mM ATP and protease inhibitors) pre-warmed at $37^{\circ} \mathrm{C}$. Then the lysates were incubated for $10 \mathrm{~min}$ at $37^{\circ} \mathrm{C}$ with shaking and centrifuged at $100,000 \mathrm{~g}$ for $1 \mathrm{~h}$ at $37^{\circ} \mathrm{C}$. The pellets containing F-actin were solubilized with actin depolymerization buffer (100 mM Pipes, $\mathrm{pH}$ 6.9, $1 \mathrm{mM}$ $\mathrm{MgCl} 2,10 \mathrm{mM} \mathrm{CaCl} 2$ and $5 \mu \mathrm{M}$ cytochalasin D) and incubated for $1 \mathrm{~h}$ on ice. The supernatant (G-actin) and pellet (F-actin) fractions were analyzed by western blotting.

\section{Cryo-electron microscopy}

For virus packaging, supernatants were collected from 293T cells in $15 \mathrm{~cm} 2$ dish transfected as described above before being centrifuged at $4000 \mathrm{rpm}$ for $5 \mathrm{~min}$ and then filtered through a $0.45 \mu \mathrm{m}$ filter to remove cell debris. Virion particles were pelleted through $20 \%$ sucrose cushion and resuspended in $100 \mu \mathrm{L}$ PBS, stored at $4{ }^{\circ} \mathrm{C}$. A $3.5 \mu \mathrm{L}$ virus sample solution was applied onto a glow discharged copper grid coated with holey carbon (R2/1; Quantifoil, Jena, Germany) prior to plunge-freezing. The grids were blotted for $3.5 \mathrm{~s}$, vitrified by plunge-freezing into liquid ethane using a Vitrobot Mark IV (Thermal Fisher Scientific). The grids were imaged using a Tecnai Arctica microscope operated at a voltage of $200 \mathrm{kV}$ equipped with a Falcon III direct electron detector (Thermal Fisher Scientific) at a nominal magnification of $39,000 \times$. Each movie consists of 40 frames was recorded at a calibrated pixel size of $2.69 \AA$, with $0.05 \mathrm{~s} /$ frame exposure, giving a total dose of $\sim 30 \mathrm{e} / \AA 2$ per movie. The electron beam induced motion was corrected by MotionCor $2^{43}$ by averaging 40 frames for each tilt. Image visualization and analysis were performed in $\mathrm{IMOD}^{44}$.

\section{Statistical analysis}

All experiments have repeated at least three times unless otherwise specified. The bar graphs were shown with mean $\pm \mathrm{SD}$. Unpaired two-tailed $t$-test is used to calculate the $p$-value unless otherwise specified.

\footnotetext{
Acknowledgements

This work was supported by China National Funds for Excellent Young Scientists (31722030) to X.T. and grants from Beijing Advanced Innovation Center for Structural Biology, Beijing Frontier Research Center for Biological Structure to X.T. and S.L. We thank Paul Spearman for reagents and discussion and Feng Zhou for help with the illustration.
}

\section{Author details}

${ }^{1}$ Beijing Advanced Innovation Center for Structural Biology, Beijing Frontier Research Center for Biological Structure, MOE Key Laboratory of Bioorganic Phosphorus Chemistry \& Chemical Biology, School of Pharmaceutical Sciences, Center for Infectious Disease Research, School of Medicine, Tsinghua

University, Beijing 100084, China. ${ }^{2}$ Beijing Advanced Innovation Center for Structural Biology, Beijing Frontier Research Center for Biological Structure, Tsinghua-Peking Center for Life Sciences, School of Life Sciences, Tsinghua University, Beijing 100084, China. ${ }^{3}$ Center for Plant Biology, School of Life Sciences, Tsinghua University, Beijing 100084, China 


\section{Author contributions}

X.T. conceived and supervised the project; Y.L. conducted most of the experiments with the help from S.Z;; Y.S. and S.L. conducted Cryo-EM analysis; M.D. and S.H. contributed reagents and expertise on the in vitro actin experiments. X.T. and Y.L. wrote the manuscript.

\section{Conflict of interest}

The authors declare that they have no conflict of interest.

\section{Publisher's note}

Springer Nature remains neutral with regard to jurisdictional claims in published maps and institutional affiliations.

Supplementary Information accompanies the paper at (https://doi.org/ 10.1038/s41421-020-0184-9).

Received: 8 April 2020 Accepted: 18 May 2020

Published online: 04 August 2020

\section{References}

1. Malim, M. H. \& Bieniasz, P. D. HIV restriction factors and mechanisms of evasion. Cold Spring Harb. Perspect. Med. 2, a006940 (2012).

2. Yan, N. \& Chen, Z. J. Intrinsic antiviral immunity. Nat. Immunol. 13, 214-222 (2012).

3. Towers, G. J. \& Noursadeghi, M. Interactions between HIV-1 and the cellautonomous innate immune system. Cell Host Microbe 16, 10-18 (2014).

4. Altfeld, M. \& Gale, M. Jr. Innate immunity against HIV-1 infection. Nat. Immunol. 16, 554-562 (2015).

5. Simon, V., Bloch, N. \& Landau, N. R. Intrinsic host restrictions to HIV-1 and mechanisms of viral escape. Nat. Immunol. 16, 546-553 (2015).

6. Harris, R. S., Hultquist, J. F. \& Evans, D. T. The restriction factors of human immunodeficiency virus. J. Biol. Chem. 287, 40875-40883 (2012).

7. Hotter, D. \& Kirchhoff, F. Interferons and beyond: induction of antiretroviral restriction factors. J. Leukoc. Biol. 103, 465-477 (2018).

8. Sauter, D. \& Kirchhoff, F. Multilayered and versatile inhibition of cellular antiviral factors by HIV and SIV accessory proteins. Cytokine Growth Factor Rev. 40, 3-12 (2018).

9. Mahon, C., Krogan, N. J., Craik, C. S. \& Pick, E. Cullin E3 ligases and their rewiring by viral factors. Biomolecules 4, 897-930 (2014).

10. Liu, Y. et al. Proteomic profiling of HIV-1 infection of human $\mathrm{CD}^{+}{ }^{+} \mathrm{T}$ cells identifies PSGL-1 as an HIV restriction factor. Nat. Microbiol. 4, 813-825 (2019).

11. Rihn, S. J. et al. The Envelope Gene of Transmitted HIV-1 Resists a Late Interferon Gamma-Induced Block. J. Virol. 91, e02254-16 (2017).

12. Carlow, D. A. et al. PSGL-1 function in immunity and steady state homeostasis. Immunol. Rev. 230, 75-96 (2009).

13. Zarbock, A., Ley, K., McEver, R. P. \& Hidalgo, A. Leukocyte ligands for endothelial selectins: specialized glycoconjugates that mediate rolling and signaling under flow. Blood 118, 6743-6751 (2011).

14. Snapp, K. R., Heitzig, C. E. \& Kansas, G. S. Attachment of the PSGL-1 cytoplasmic domain to the actin cytoskeleton is essential for leukocyte rolling on Pselectin. Blood 99, 4494-4502 (2002).

15. Serrador, J. M. et al. A juxta-membrane amino acid sequence of P-selectin glycoprotein ligand-1 is involved in moesin binding and ezrin/radixin/moesindirected targeting at the trailing edge of migrating lymphocytes. Eur. J. Immunol. 32, 1560-1566 (2002).

16. Kanellos, G. \& Frame, M. C. Cellular functions of the ADF/cofilin family at a glance. J. Cell Sci. 129, 3211-3218 (2016).

17. Bukrinskaya, A., Brichacek, B., Mann, A. \& Stevenson, M. Establishment of a functional human immunodeficiency virus type $1(\mathrm{HIV}-1)$ reverse transcription complex involves the cytoskeleton. J. Exp. Med. 188, 2113-2125 (1998).

18. Yoder, A. et al. HIV envelope-CXCR4 signaling activates cofilin to overcome cortical actin restriction in resting CD4 T cells. Cell 134, 782-792 (2008).
19. Chazotte, B. Labeling cytoskeletal F-actin with rhodamine phalloidin or fluorescein phalloidin for imaging. Cold Spring Harb. Protoc. 2010, pdb. prot4947 (2010)

20. Riedl, J. et al. Lifeact: a versatile marker to visualize F-actin. Nat. Methods $\mathbf{5}$, 605-607 (2008)

21. Nishita, M., Aizawa, H. \& Mizuno, K. Stromal cell-derived factor 1alpha activates LIM kinase 1 and induces cofilin phosphorylation for T-cell chemotaxis. Mol. Cell. Biol. 22, 774-783 (2002).

22. Snapp, K. R. et al. A novel P-selectin glycoprotein ligand-1 monoclonal antibody recognizes an epitope within the tyrosine sulfate motif of human PSGL1 and blocks recognition of both P- and L-selectin. Blood 91, 154-164 (1998).

23. Chugh, P. \& Paluch, E. K. The actin cortex at a glance. J. Cell Sci. 131, jcs.186254 (2018).

24. Zheng, Y., Xie, Y., Jiang, Y., Qu, X. \& Huang, S. Arabidopsis actin-depolymerizing factor7 severs actin filaments and regulates actin cable turnover to promote normal pollen tube growth. Plant Cell 25, 3405-3423 (2013).

25. Cavrois, M., De Noronha, C. \& Greene, W. C. A sensitive and specific enzymebased assay detecting HIV-1 virion fusion in primary $\mathrm{T}$ lymphocytes. Nat. Biotechnol. 20, 1151-1154 (2002).

26. Linde, M. E. et al. The conserved set of host proteins incorporated into HIV-1 virions suggests a common egress pathway in multiple cell types. J. Proteome Res. 12, 2045-2054 (2013).

27. Bates, M., Jones, S. A. \& Zhuang, X. Stochastic optical reconstruction microscopy (STORM): a method for superresolution fluorescence imaging. Cold Spring Harb. Protoc. 2013, 498-520 (2013).

28. Epperson, T. K., Patel, K. D., McEver, R. P. \& Cummings, R. D. Noncovalent association of P-selectin glycoprotein ligand-1 and minimal determinants for binding to P-selectin. J. Biol. Chem. 275, 7839-7853 (2000).

29. Grover, J. R., Veatch, S. L. \& Ono, A. Basic motifs target PSGL-1, CD43, and CD44 to plasma membrane sites where HIV-1 assembles. J. Virol. 89, 454-467 (2015)

30. Kirschman, J. et al. HIV-1 envelope glycoprotein trafficking through the endosomal recycling compartment is required for particle incorporation. $J$. Virol. 92, e01893-17 (2018).

31. Taylor, M. P., Koyuncu, O. O. \& Enquist, L. W. Subversion of the actin cytoskeleton during viral infection. Nat. Rev. Microbiol. 9, 427-439 (2011).

32. Haddad, W. et al. P-selectin and P-selectin glycoprotein ligand 1 are major determinants for Th1 cell recruitment to nonlymphoid effector sites in the intestinal lamina propria. J. Exp. Med. 198, 369-377 (2003).

33. Veerman, K. M. et al. Interaction of the selectin ligand PSGL-1 with chemokines CCL21 and CCL19 facilitates efficient homing of T cells to secondary lymphoid organs. Nat. Immunol. 8, 532-539 (2007).

34. Tinoco, R. et al. PSGL-1 is an immune checkpoint regulator that promotes $T$ cell exhaustion. Immunity 44, 1470 (2016).

35. Freed, E. O. HIV-1 assembly, release and maturation. Nat. Rev. Microbiol. 13 484-496 (2015)

36. Murakami, T., Carmona, N. \& Ono, A. Virion-incorporated PSGL-1 and CD43 inhibit both cell-free infection and transinfection of HIV-1 by preventing viruscell binding. Proc. Natl Acad. Sci. USA 117, 8055-8063 (2020).

37. Fu, Y. et al. PSGL-1 restricts HIV-1 infectivity by blocking virus particle attachment to target cells. Proc. Natl. Acad. Sci. USA 117, 9537-9545 (2020).

38. Butler, S. L., Hansen, M. S. T. \& Bushman, F. D. A quantitative assay for HIV DNA integration in vivo. Nat. Med. 7, 631-634 (2001).

39. Cavrois, M., De Noronha, C. \& Greene, W. C. A sensitive and specific enzymebased assay detecting HIV-1 virion fusion in primary $\mathrm{T}$ lymphocytes. Nat. biotechnolBiotechnol 20, 1151-1154 (2002).

40. Zheng, Y., Xie, Y., Jiang, Y., Qu, X. \& Huang, S. Arabidopsis actin-depolymerizing factor7 severs actin filaments and regulates actin cable turnover to promote normal pollen tube growth. Plant Cell 25, 3405-3423 (2013).

41. Doolittle, L. K., Rosen, M. K. \& Padrick, S. B. in Adhesion Protein Protocols (ed Amanda S. Coutts) 273--293 (Humana Press, 2013).

42. Yang, S. Y. et al. Secretagogin affects insulin secretion in pancreatic á-cells by regulating actin dynamics and focal adhesion. Biochem. J. 473, 1791-1803 (2016).

43. Zheng, S. Q. et al. MotionCor2: anisotropic correction of beam-induced motion for improved cryo-electron microscopy. Nat. Methods 14, 331 (2017).

44. Kremer, J. R., Mastronarde, D. N. \& McIntosh, J. R. Computer visualization of three-dimensional image data using IMOD. J. Struct. Biol. 116, 71-76 (1996). 